\title{
Leucocytoclastic Vasculitis: A Rare Cause of Palpable Purpura
}

\author{
APARNA DAS, ${ }^{1}$ AKM AMINUL HOQUE, ${ }^{2}$ RATAN DAS GUPTA, ${ }^{3}$ GOBINDA BANIK, ${ }^{4}$ MD. YEANUR HOSSAIN, ${ }^{5}$ \\ MOINUL ISLAM ${ }^{6}$
}

\begin{abstract}
:
Leucocytoclastic vasculitis (LCV) is an isolated condition without systemic vasculitis or glomerulonephritis . The skin is the most commonly involved organ in LCV, predominantly in the lower extremities. The most common skin manifestation is palpable purpura. Some patients may have arthralgias or arthritis as the presenting symptom, usually oligoarthritis of the knees or ankles. The inflammation of small blood vessels, most commonly postcapillary venules, is the cardinal histologic feature of LCV. A I6-year-old male admitted into our medicine unit with palpable purpura. He was diagnosed as leukocytoclastic vasculitisof unidentified etiology on the basis of clinical conditions, laboratory tests, and histopathological analysis. Most important cause of LCV is drugs. So, we report this case to increase the awareness of indiscriminate use of medicine which can case this problems.
\end{abstract}

Keyword: leukocytoclastic vasculitis, Palpable purpura, Drugs

\section{Introduction:}

The term leukocytoclastic vasculitis (LCV) has been termed as small- vessel vasculitides, including drug-induced vasculitis, allergic vasculitis, hypersensitivity angiitis, and hypersensitivity vasculitis (HSV). ${ }^{1}$ The American College of Rheumatology (ACR) has developed criteria for the classification of hypersensitivy vasculitis. ${ }^{1,2}$ American College of Rheumatology Criteria for Hypersensitivity Vasculitis : 1. Age $>16$ years at disease onset, 2.Medication at disease onset as precipitating factor, 3.Palpable purpura, 4.Maculopapular rash, 5. Skin biopsy with granulocytes around an arteriole or venule. For $>3$ criteria: sensitivity $71 \%$ and specificity $84 \%$ for the diagnosis of HSV.When used diagnostically the positive predictive value of these criteria is only $\sim 30 \%$. In addition, other limitations include the lack of differentiation of hypersensitivity vasculitis from Henoch-Schönlein purpura (HSP). The inflammation of small blood vessels, most commonly postcapillary venules, is the cardinal histologic feature of $\mathrm{LCV} .{ }^{1}$ Other characteristic features include fibrinoid necrosis of the vessel walls, leukocytoclasis, and hemorrhage. ${ }^{1,3}$ The inflammatory infiltrate is typically neutrophilic; other studies have shown

1. Assistant. Prof., Department of Medicine. DMCH

2. Associate. Prof., Department of Medicine .DMCH

3. Assistant. Prof., Department of Nephrology .DMC

4. Lecturer, Department of Harmatology, SSMC

5. Registrar, Department of Medicine. DMCH

6. Assistant.Registrar, Department of Medicine. DMC

Correspondence: Dr. Aparna Das. Assistant. Prof. Department of Medicine. DMCH a predominance of mononuclear cells and eosinophils distributed in all vessel layers. Direct immunofluorescence has been detected in LCV, in which early stages of vascular injury have fibrinogen, $\mathrm{C} 3$, and immunoglobulin $\mathrm{M}$ deposits have been detected in the vessel wall. ${ }^{1}$ In fully manifest lesions, albumin, fibrinogen, and IgG deposits are present; lesions at later stages have fibrinogen and $\mathrm{C} 3$ deposited in vessel walls. ${ }^{1,4}$ The severity of the lesion may correlate with disease course.

\section{Case report:}

A 16-year-old male was admitted into our medicine unit with rashes that persisted for the last 30 days. At first, rashes were painful, erythematous and macular involving both lower limbs. In a short period of time (around 24 hours) some lesions became ulcerated. Rashes subsequently involving the both upper limbs sparing palms, sole and buttock. Rashes are associated with arthralgia of both knees, ankles and small joints of hands. Rashes had slow and spontaneous healing without scarring. On initial physical examination the patient having reddish, purpuric patches and macules that were palpable, non-blanchable and tender lesions distributed bilaterally over both legs and both forearms. These lesions were consistent with vasculitis. There was a ulcer on the lateral aspect of the right leg. Features of arthritis were present in both knees and only tenderness present in both wrist and small joint of hands.

Laboratory findings revealed ESR (erythrocyte sedimentation rate) normal $(40 \mathrm{~mm} / \mathrm{h})$. The $\mathrm{C}$-reactive protein test $+v e$, which indicates acute inflammation, and the $\mathrm{C} 3$ and $\mathrm{C} 4$ complement components were within normal levels. ANF (antinuclear 


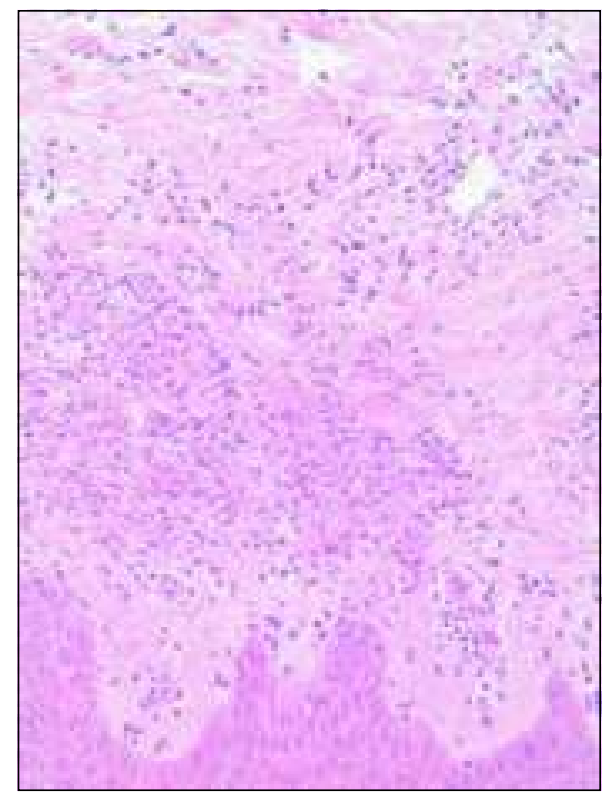

Fig.-1:

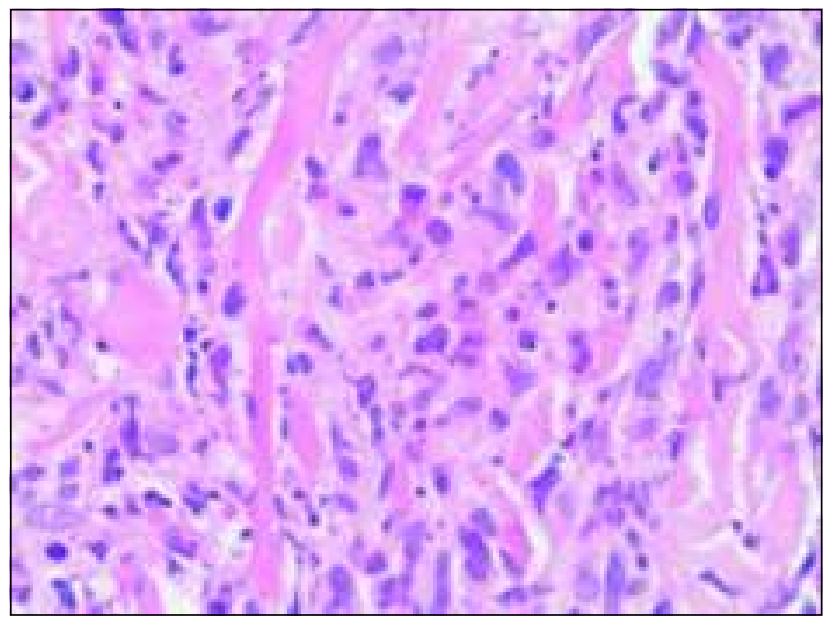

Fig.-2:

factor), RA, Anti CCP Antibody and ANCA were unrevealing. Image likeCXR, ECG and USG of abdomen were normal. Echocardiogram revealed minimal pericardial effusion. The skin biopsy revealed mild acanthosis in epidermis. The dermal vessels show swelling of endothelial cells and infiltrate of polymorphs and lymphocyte admixed with karyorrhectic debris. Direct immune fluroscence study showed deposition of $\operatorname{IgA}, \operatorname{IgG}, \operatorname{IgM}, \mathrm{C}_{3}$ or fibrinogen which was suggestive of leucocytoclastic vasculitis.

\section{Discussion :}

Clinically, the leukocytoclastic vasculitis is different from thrombocytopenia, trauma or vascular vessels walls disorders by the presence of palpable purpuric eruptions. The skin is the most commonly involved organ in LCV, predominantly in the lower extremities. ${ }^{1,6,7} \mathrm{Up}$ to one-third of patients have trunk and upper extremity involvement, typically sparing the palmar, plantar, and mucosal surfaces .In our patient ,rashes first appear in lower extrimities which was palpable and subsequently involve the upper extrimities. The most common skin manifestation and the most sensitive finding of HSV is palpable purpura. Other skin manifestations include maculopapular rash, bullae, papules, plaques, nodules, ulcers, and livedo reticularis .A few rashes were ulcerated in legs. Other manifestations of LCV include joint involvement .Some patients may have arthralgias or arthritis as the presenting symptom, usually oligoarthritis of the knees or ankles . Our patient also had oligoarthritis, arthralgia and fever. Systemic manifestations of LCV are much less common than its dermatologic features. These include fever, microscopic hematuria, elevated creatinine, pericarditis and pleuritis. ${ }^{1,8}$

Numerous series and case reports have reported drugs as the precipitating agent in LCV. ${ }^{1,7,9}$ In patients with biopsy proven LCV, $24 \%$ had drug exposure within a week of presentation. ${ }^{1,6}$ There are many drugs that have been implicated in the development of LCV including antibiotics, non-steroidal anti-inflammatory drugs (NSAIDs), methotrexate, azathioprine, etanercept, cyclosporine, allopurinol, sulfasalazine, gold salts, antithyroid agents, anticonvulsants, antiarrhythmics, and diuretics. No cause was found in case of our patient. There aren't specific laboratory tests to detect the leukocytoclastic vasculitis, they only aid the evaluation of the organic functions (blood investigations, screening tests for inflammatory diseases, levels of serum complement, serological tests for hepatitis B and $\mathrm{C}$ and herpes, ANCA, antiphospholipid antibodies, urinalysis and Chest Xray).$^{10,11,12}$ The skin biopsy has a paramount role in the diagnosis of this illness, because it allows for the assessment of the damaged vessel caliber. It also makes possible to distinguish the leukocytoclastic vasculitis from other non-inflammatory vasculopathies that emulate it, like perivasculitis, embolic phenomena, and cryoglobulinemia. Biopsy should be early performed once leukocytoclasia may not be found in old lesions, which can present a predominance of lymphocytes around blood vessels. The histopathologic test reveals an angiocentric inflammatory process associated with leukocytoclasia (neutrophil segmentation), edema of endothelial cells, extravasation of red blood cells and fibrinoid necrosis. The presence of immunoglobulins in lesions may be observed by immunofluorescence; however, their presence in vascular lesions is rare and does not justify the routine use of this test ${ }^{1}$. 
Most cases of LCV are self-limited, lasting several weeks to months. ${ }^{9}$ One study showed that $90 \%$ of patients with HSV had resolution of symptoms in less than one year ${ }^{1,8}$. Also, most patients with LCV have complete recovery without sequelae. ${ }^{1,6}$ Most patients with an acute episode of HSV do not require treatment. Patients with an identifiable precipitating etiology have outcomes similar to that of patients without a known trigger. ${ }^{7}$ Patients with palpable purpura have a better disease outcome and course, as compared to patients with vasculitic skin ulcers.

The treatment of HSV includes discontinuing any causative medication. If the identification of a single drug is not possible then there should be an attempt to withdraw as many suspected etiologies as possible agents involved in the treatment of LCV are NSAIDs, colchicine, dapsone, corticosteroids, cyclophosphamide, azathioprine, plasma exchange, and intravenous immune globulin. ${ }^{1}$

In the case reported here, signs, clinical symptoms, and tests findings were in accordance with the data of the American College of Rheumatology. This provided us with the appropriate conditions to define the diagnosis of our patient as leukocytoclastic vasculitis. Though one of the most important causes of leukocytoclastic vasculitis is drugs but most of drugs are found in our country as over the counter drugs. So this case report enlightens the people and physicians about the uses of medicine including herbal and homeopathy.

\section{Conflict of Interest: None}

\section{References:}

1. Daniel CL: Leukocytoclastic Vasculitis and HenochSchönlein Purpura. Arthritis \& Allied Conditions 15th edition. 2005; 86: 1793-1797
2. Calabrese LH, Michel BA, Bloch DA, Arend WP, Edworthy SM, Fauci AS, Fries JF, Hunder GG, Leavitt RY, Lie JT: The American College of Rheumatology 1990 criteria for the classification of hypersensitivity vasculitis. Arthritis Rheum 1990; 33(8): 1108-1113

3. Sams WM: Hypersensitivity angiitis. J Invest Dermatol 1989; 93: 785-815

4. Grunwald MH, Avinoach I, Amichai B, Halevy S: Leukocytoclastic vasculitis: correlation between different histologic stages and direct immunofluorescence results. Int J Dermatol 1997; 37: 349-352

5. Ratnam KV, Boon YH, Pank BK: Idiopathic hypersensitivity vasculitis: clinicopathologic correlation of 61 cases. Int $\mathbf{J}$ Dermatol 1995; 34: 786-789

6. García-Porrúa C, Gonzalez-Gay MA, López-Lázaro L: Drug associated cutaneous vasculitis in adults in northwestern Spain. J Rheumatol 1999; 26: 1942-1944

7. Martinez-Taboada VM, Blanco R, Garcia-Fuentes M, Rodriguez-Valverde V: Clinical features and outcome of 95 patients with hypersensitivity vasculitis. Am J Med 1997; 102: 186-191

8. Taylor HG, Samanta A: Treatment of Vasculitis. Brit J Clin Pharmacol 1993; 35: 93-104

9. Del Pozo, J, Sacristán F, Martínez W, Paradela S, FernándezJorge B, Fonseca E: Neutrophilic dermatosis of the hands: presentation of eight cases and review of the literature. $\mathrm{J}$ Dermatol 2007; 34: 243-247

10. Millikan LE, Flynn T. Infectious etiologies of cutaneous vasculitis. Clin Dermat 1999; 17: 509-14

11. Amer M, Metwalli M. Cutaneous vasculitis in Western Africa. Clin Dermat 1999; 17: 655-8

12. Fiorentino DF. Cutaneous vasculitis. J Am Ac Dermatol 2003; 48: 311-40 